As an example, let us show the reconstruction of the three-electrode electrostatic lens from the optimized axial potential distribution given in Ref. 4. The electrode shapes and potentials are given in Fig. 1. The axial potential distribution of this system is close to that of the original theoretical distribution (the maximum discrepancy is $8.3 \%$ of the maximum value of the potential). The calculated value of the spherical aberration coefficient $C_{\text {so }}$ referred to the object side and related to the object side focal length $f_{o}$ is $C_{s o} / f_{o}=0.95$ as opposed to $C_{s o} / f_{o}=1.02$ for the theoretical distribution. The reconstruction was carried out on a VAX 11/780 computer and required $1.5 \mathrm{~s}$ of CPU time.

This work is supported by the National Science Foundation, Solid State and Microstructures Engineering grant No. ECS 8317485 . The author is indebted to Dr. Gregory
Baker for a valuable discussion. The assistance of Mr. Mark Beardslee in the computational work is appreciated.

${ }^{1}$ W. Tretner, Optik 16, 155 (1959).

${ }^{2}$ R. W. Moses, Rev. Sci. Instrum. 42, 828 (1971)

${ }^{3}$ M. Szilagyi, Optik 48, 215 (1977).

${ }^{4}$ M. Szilagyi, S. J. Yakowitz, and M. O. Duff, Appl. Phys. Lett. 44, 1, 7 (1984).

${ }^{5}$ W. Glaser, Grundlagen der Elektronenoptik (Foundations of Electron Optics, Springer, Wien, 1952).

${ }^{6}$ O. Scherzer, Z. Phys. 101, 23 (1936)

${ }^{7}$ G. N. Plass, J. Appl. Phys. 13, 582 (1942).

${ }^{8}$ M. Szilagyi, Electron Microscopy, Proc. 7th European Congress 1, 62 (1980).

${ }^{9}$ P. W. Hawkes, J. Phys. E 14, 1353 (1981).

${ }^{10} \mathrm{~J}$. W. Butler, in Proceedings of the 6th International Congress for Electron Microscopy, Kyoto, 1966, p. 191.

${ }^{11}$ M. Szilagyi, J. Vac. Sci. Technol. B 1, 1137 (1983).

\title{
Detuned loading in coupled cavity semiconductor lasers-Effect on quantum noise and dynamics
}

\author{
Kerry Vahala and Amnon Yariv \\ California Institute of Technology, Pasadena, California 91125
}

(Received 5 March 1984; accepted for publication 21 May 1984)

\begin{abstract}
We derive the modulation and noise properties of a semiconductor laser consisting of an active cavity loaded by a passive cavity. The results indicate that under certain conditions the direct modulation bandwidth can be doubled with simultaneous phase noise reduction as compared to a conventional laser.
\end{abstract}

The subject of coupled cavity semiconductor lasers [e.g., cleaved coupled cavity $\left(\mathbf{C}^{3}\right),{ }^{1}$ grin rod external coupled cavity (GRECC) $\left.{ }^{2}\right]$ has experienced renewed interest as a means of achieving superior modal selectivity. Very little effort, however, has been directed toward a fundamental understanding of the modulation dynamics and quantum noise properties of these devices. In a conventional semiconductor laser two important quantities characterize modulation and phase noise: the relaxation oscillation frequency $v_{R}$ sets the useful direct modulation bandwidth of the device, ${ }^{3}$ and the linewidth enhancement factor $\alpha$ (or amplitude phase coupling factor) determines both the ratio of FM to AM modulation indices in the output of a directly modulated laser ${ }^{4}$ as well as the degree to which spectral purity (as determined by linewidth $\Delta v$ of the lasing mode) is degraded by amplitude phase coupling. ${ }^{5-8}$ Analyses given elsewhere ${ }^{3,4,7}$ have shown that the conventional (uncoupled) expressions for these quantities are of the form

$$
\begin{aligned}
& v_{R}^{2}=\left[1 /(2 \pi)^{2}\right]\left(g^{\prime} P / \tau\right), \\
& \alpha=\left(2 \bar{\omega} / \mu_{0}\right)\left(\mu^{\prime} / g^{\prime}\right),
\end{aligned}
$$

where the linewidth and the FM/AM indice ratio are given by

$$
\begin{aligned}
& \Delta v=\Delta v_{\mathrm{ST}}\left(1+\alpha^{2}\right), \\
& \dot{\varphi} / \dot{\rho}=-\alpha .
\end{aligned}
$$

In these expressions $g^{\prime}\left(\mu^{\prime}\right)$ is the derivative of gain (resonant refractive index) with respect to carrier density; $P, \bar{\omega}$, and $\tau$ are the photon density, lasing frequency, and unpumped photon lifetime of the lasing mode; $\Delta v_{\mathrm{ST}}$ is the modified Schawlow-Townes linewidth formula; ${ }^{7} \mu_{0}$ is the nonresonant contribution to refractive index; and $\varphi$ and $\rho$ are the small-signal phase and amplitude variations of the lasing field. In this letter we will derive the modulation corner frequency and the effective amplitude phase coupling parameter of an active cavity/passive cavity compound semiconductor laser. We show that because of physical effects unique to lasing action in a semiconductor (a strongly detuned gain spectrum leading to amplitude phase coupling of the lasing field $^{9}$ ) several major improvements in the modulation and noise performance can be expected to result in a coupled cavity laser. These include the following simultaneous improvements over conventional (uncoupled) lasers: first, enhancement of the direct modulation corner frequency $v_{R}$ by nearly $2 \times$; second, suppression of the FM component of modulation with respect to the AM component; third, lasing linewidth reduction (i.e., phase noise reduction). The third improvement given here has been predicted and observed in semiconductor lasers subjected to small amounts of optical feedback. ${ }^{10-12}$

The physical mechanism responsible for the aforemen- 
tioned improvements is actually more general than the coupled cavity context under which it is presented here. It involves the introduction of any dispersive loss mechanism into the laser followed by deliberate detuning of the lasing frequency away from the loss peak (hence the name "detuned loading"). This more general picture, described in detail elsewhere, ${ }^{13}$ predicts that the same improvements should also occur in semiconductor lasers with distributed feedback. For the purposes of this discussion, however, we consider a specific model consisting of two cavities (one active, one passive), and begin by writing the coupled equations of motion for the complex field amplitudes in each resonator.

$$
\begin{aligned}
& \dot{E}_{m}=\left(\iota \omega_{m}-1 / 2 \tau_{m}\right) E_{m}+f_{1} E \\
& \dot{E}=\left(\iota \omega-\frac{1}{2 \tau}\right) E+f_{2} E_{m}-\iota \frac{\Gamma}{2}\left(\frac{2 \omega}{\mu_{0}} \mu(n)+\iota g(n)\right) E
\end{aligned}
$$

Neglecting for a moment the coupling terms involving $f_{1}$ and $f_{2}$, Eq. (5) is simply the damped equation of motion for the $m$ th mode of the passive resonator $\left(\omega_{m}\right.$ and $\tau_{m}$ are the frequency and the photon lifetime associated with this mode). Equation (6) is the driven equation of motion for the lasing field amplitude $E$; this field is the result of stimulated emission into the cavity mode having a passive resonant frequency $\omega$ and photon lifetime $\tau$. Incomplete spatial overlap of the lasing mode and the active medium [assumed to be spatially uniform with gain $g(n)$ and resonant refractive index $\mu(n)$ which depend on the carrier density $n$ ] is accounted for by the filling factor $\Gamma$. It is assumed that the field variables are normalized so that $|E|^{2} \equiv P$ is an average photon density. For simplicity, we assume the characteristics of each resonator are chosen so that only one mode from each resonator couples well to a single mode from the other resonator (although the more general situation can be easily accommodated). A detailed justification of forms (5) and (6), including a discussion of the properties of the coupling rate parameters $f_{1}$ and $f_{2}$, will be given in a more comprehensive treatment. ${ }^{13}$ In addition to the field equations (5) and (6), it is also necessary to consider the interaction of the carrier density with the field. The result of this analysis, however, will be to replace $g^{\prime}, \mu^{\prime}$, and $\tau$ in (1) and (2) with new effective quantities, and for this reason it is unnecessary to include a carrier density rate equation in the analysis. It will be seen that the amplitude phase coupling parameter $\alpha$ [typically in the range -4 to -6 (Refs. 6,7$)$ ] weighs heavily in determining the size of these new quantities, leading to several predictions of considerable importance.

As a first step towards solving (5) and (6) we represent the lasing field amplitude in the active resonator in terms of slowly varying amplitude and phase components as follows:

$$
E(t)=E_{0}[1+\rho(t)] e^{i(\bar{\omega} t+\varphi(t))} .
$$

Using this form, (5) can be integrated to yield

$E_{m}(t)=f_{1} A_{0} e^{i \bar{\omega} t} \int_{-\infty}^{t} e^{\left[\iota\left(\omega_{m}-\bar{\omega}\right)-1 / 2 \tau_{m}\right]^{(t-z)}}[1+\rho(z)] e^{\imath \varphi(z)} d z$

The slowly varying amplitude and phase terms $\rho(t)$ and $\varphi(t)$ vary on a nanosecond timescale in semiconductor lasers ${ }^{8}$; the lifetime $\tau_{m}$ in (8), however, is typically a few picoseconds (for small passive resonators). This allows us to expand the slowly varying quantities appearing in (8) to first order in the variable $(t-z)$. Performing the integration after this expansion gives

$$
\begin{aligned}
E_{m}(t) \approx & \frac{f_{1} E(t)}{\iota\left(\bar{\omega}-\omega_{m}\right)+1 / 2 \tau_{m}} \\
& -\frac{f_{1} E_{0}[\dot{\rho}(t)+\iota \dot{\varphi}(t)] e^{\iota[\bar{\omega} t+\varphi(t) \mid}}{\left[\iota\left(\bar{\omega}-\omega_{m}\right)+1 / 2 \tau_{m}\right]^{2}} .
\end{aligned}
$$

This expression allows Eq. (6) to be rewritten in terms of variables pertaining only to the field amplitude $E(t)$. The equation which results from this substitution is now linearized about a steady state operating point using (7) and the following first order Taylor expansions of gain and resonant refractive index about the operating point,

$$
\begin{aligned}
& g(n) \approx g\left(n_{0}\right)+g^{\prime} n_{1}, \\
& \mu(n) \approx \mu\left(n_{0}\right)+\mu^{\prime} n_{1},
\end{aligned}
$$

where we take $n_{1}$ as the small-signal carrier density change about its operating point value $n_{0}$. The time averaged equations which in part establish the operating point are (a complete set of operating point equations would also include a steady state form of the carrier density rate equation)

$$
\begin{aligned}
& \Gamma g\left(n_{0}\right)=\frac{1}{\tau}+\frac{1}{\tau_{m}} \frac{\gamma}{1+\Delta^{2}} \equiv \frac{1}{\tau_{\text {eff }}}, \\
& \Delta\left[1-\frac{\gamma}{1+\Delta^{2}}\right]=\delta
\end{aligned}
$$

with a tuning parameter given by

$$
\delta \equiv 2 \tau_{m}\left[\omega-\omega_{m}-\frac{\Gamma \omega}{\mu_{0}} \mu\left(n_{0}\right)\right],
$$

and where $\gamma \equiv-4 \tau_{m}^{2} f_{1} f_{2}$ is the coupling strength parameter, and $\Delta$ is defined in terms of the actual lasing frequency $\bar{\omega}$ by $\bar{\omega} \equiv \omega_{m}+\Delta / 2 \tau_{m}$. Equation (12) gives the threshold gain for a lasing eigenfrequency $\bar{\omega}$ and therefore also the effective loss rate $\tau_{\text {eff }}^{-1}$ for eigenmodes of the composite cavity. Equation (13) gives the possible lasing eigenfrequencies (in terms of $\Delta$ ) as a function of the tuning parameter $\delta$ and the coupling strength $\gamma$.

The small-signal equations which result from the linearization procedure appear below:

$$
\begin{aligned}
& \left(1+\beta_{2}\right) \dot{\rho}+\beta_{1} \dot{\varphi}=\left(\Gamma g^{\prime} / 2\right) n_{1}, \\
& -\beta_{1} \dot{\rho}+\left(1+\beta_{2}\right) \dot{\varphi}=-\left(\Gamma \omega / \mu_{0}\right) \mu^{\prime} n_{1},
\end{aligned}
$$

where

$$
\begin{aligned}
& \beta_{1} \equiv-2 \gamma \Delta /\left(1+\Delta^{2}\right)^{2}, \\
& \beta_{2} \equiv-\gamma\left(1-\Delta^{2}\right) /\left(1+\Delta^{2}\right)^{2} .
\end{aligned}
$$

Simple algebraic rearrangement of (15) and (16) yields

$$
\begin{aligned}
& \dot{\rho}=(\Gamma / 2) g_{\text {eff }}^{\prime} n_{1}, \\
& \dot{\varphi}=-\left(\Gamma \omega / \mu_{0}\right) \mu_{\text {eff }}^{\prime} n_{1} .
\end{aligned}
$$

These equations have the form of the conventional dynamic equations $^{8}$ [i.e., those found by setting $\beta_{1}=0=\beta_{2}$ in (15) and (16)] with new effective differential gain and differential 


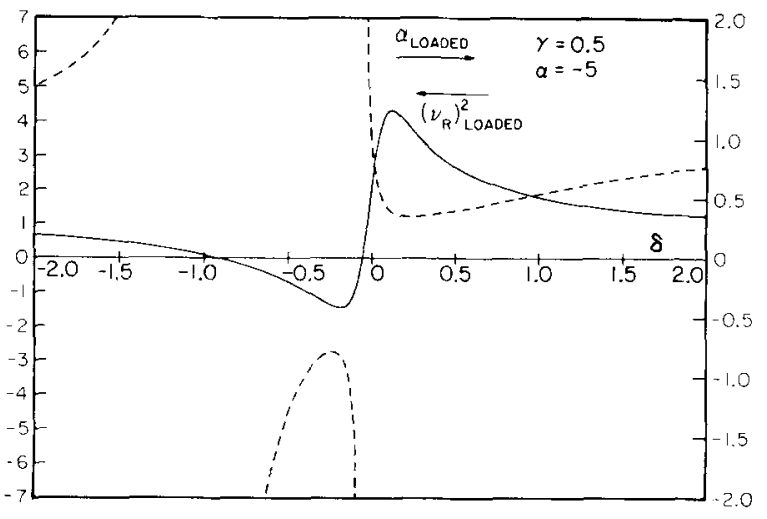

FIG. 1. Amplitude phase coupling factor (dashed curve) and square of modulation corner frequency (solid curve) normalized by their conventional (uncoupled) values and plotted vs the tuning parameter.

refractive index terms defined as

$$
\begin{aligned}
& g_{\text {eff }}^{\prime} \equiv \frac{1+\beta_{2}+\alpha \beta_{1}}{\left(1+\beta_{2}\right)^{2}+\beta_{1}^{2}} g^{\prime}, \\
& \mu_{\text {eff }}^{\prime} \equiv \frac{1+\beta_{2}-(1 / \alpha) \beta_{1}}{\left(1+\beta_{2}\right)^{2}+\beta_{1}^{2}} \mu^{\prime} .
\end{aligned}
$$

The net effect of loading by the passive resonator is to cause "mixing" of $g$ ' and $\mu$ ', thus creating new effective differential quantities. To solve for the modulation characteristics of the loaded laser we can either solve (15), (16), and a linearized carrier density rate equation, or equivalently and more simply, use the new effective quantities defined by (12), (21), and (22) in the expressions (1) and (2) for the modulation corner frequency $v_{R}$ and the amplitude phase coupling factor $\alpha$. The result is (note: the actual coupled cavity form for the linewidth $\Delta v$ is a slightly modified version of $(3)^{13} ;(1),(2)$, and (4) remain unchanged, however)

$$
\begin{aligned}
& \left(v_{R}\right)_{\text {loaded }}^{2}=\frac{1}{(2 \pi)^{2}} \frac{g_{\text {eff }}^{\prime} P}{\tau_{\text {eff }}}, \\
& \alpha_{\text {loaded }}=\frac{2 \bar{\omega}}{\mu_{0}} \frac{\mu_{\mathrm{eff}}^{\prime}}{g_{\mathrm{eff}}^{\prime}},
\end{aligned}
$$

$\left(v_{R}\right)_{\text {loaded }}^{2}$ and $\alpha_{\text {loaded }}$, normalized by their unloaded values, are plotted in Fig. 1 versus the tuning parameter $\delta$ for $\gamma=0.5$ and $\alpha=-5{ }^{6,7}$ The coupling strength $\gamma$ assumed here is easily achieved with a passive resonator only slightly larger in optical length than the active resonator. ${ }^{13}$ Negative $\gamma$ 's which from (12), are more desirable with regards to maintaining oscillation near a passive resonance, lead to similar results and are achievable in $\mathrm{C}^{3}$ devices having appropriate gap separation. This plot indicates that, under slight detuning, direct modulation bandwidth enhancement of $2 \times, F M$ suppression, and phase noise reduction are possible simultaneously. All of these improvements result from the mixing of differential quantities mentioned earlier. Specifically, the fact that $|\alpha|$ is significantly larger than unity permits an enhancement of $g_{\text {eff }}^{\prime}$ at the expense of $\mu_{\text {eff }}^{\prime}$, thus increasing modulation bandwidth and suppressing FM components of modulation as well as phase noise (note: the converse is also possible). In plotting $\left(v_{R}\right)_{\text {loaded }}^{2}$ we have neglected the $\tau_{\text {eff }}$ dependence so as not to mask the variations of the effective differential gain. Another interesting prediction of this mod$\mathrm{el}$ is a region of negative effective differential gain which appears in the plot as a tuning region of negative $\left(v_{R}\right)_{\text {loaded }}^{2}$. This is a dynamically unstable region, similar to the region of negative differential resistance in a tunnel diode or Gunn device. We will consider this region in greater detail elsewhere. ${ }^{13}$

In conclusion, we have analyzed the modulation and noise performance of a coupled cavity semiconductor laser by deriving effective differential quantities. The results suggest that significant improvements in performance over conventional (uncoupled) lasers are possible in detuned configurations of these devices. One improvement, the reduction of phase noise predicted by this treatment and others, ${ }^{10-12}$ has, in fact, been observed. ${ }^{12}$

This work was supported by the Office of Naval Research and ITT Corporation. One author (KJV) is grateful for a fellowship from IBM.

'W. T. Tsang, N. A. Olsson, and R. A. Logan, Appl. Phys. Lett. 42, 650 (1983).

${ }^{2}$ K. Y. Lious, C. A. Burrus, S. W. Grandlund, C. B. Swan, R. A. Linke, I. Kaminow, and P. Besomi, Topical Meeting on Optical Fiber Communication, New Orleans 1984, paper TuL2.

${ }^{3}$ K. Y. Lau, N. Bar Chaim, I. Ury, Ch. Harder, and A. Yariv, Appl. Phys. Lett. 43, 1 (1983).

${ }^{4}$ Ch. Harder, K. Vahala, and A. Yariv, Appl. Phys. Lett. 42, 328 (1983).

${ }^{5}$ M. Fleming and A. Mooradian, Appl. Phys. Lett. 38, 511 (1981).

${ }^{6}$ C. H. Henry, IEEE J. Quantum Electron. QE-18, 259 (1982).

${ }^{7}$ K. Vahala and A. Yariv, IEEE J. Quantum Electron. QE-19, 1096 (1983).

${ }^{8} \mathrm{~K}$. Vahala and A. Yariv, IEEE J. Quantum Electron. QE-19, 1102 (1983).

${ }^{9}$ K. Vahala, L. C. Chiu, S. Margalit, and A. Yariv. Appl. Phys. Lett, 42, 631 (1983).

${ }^{10} \mathrm{~K}$. Kikuchi, T. Okoshi, and H. Ishigami, Rec. Opt. Quantum Electron. IECE Jpn. 1983, paper OQE83-23, pp. 25-32.

"E. Patzak, H. Oleson, A. Sugimura, A. Saito, and T. Mukai, Electron. Lett. 19, 938 (1983).

${ }^{12}$ Y. C. Chen, Appl. Phys. Lett. 44, 10 (1984).

${ }^{13}$ K. Vahala, J. Paslaski, and A. Yariv (unpublished). 